\title{
FITOSSOCIOLOGIA EM COMUNIDADES ARBÓREAS REMANESCENTES DE CERRADO SENSU STRICTO NO BRASIL CENTRAL
}

\author{
Zenesio Finger $^{1 *}$, Felipe Augusto Finger ${ }^{2}$ \\ ${ }^{1 *}$ Universidade Federal do Mato Grosso, Faculdade de Engenharia Florestal, Cuiabá, Mato Grosso, Brasil - fingerz@terra.com.br \\ ${ }^{2}$ Instituto Brasileiro do Meio Ambiente e dos Recursos Naturais Renováveis, Cuiabá, Mato Grosso, Brasil - felipe.finger@ibama.gov.br
}

Recebido para publicação: 18/02/2013 - Aceito para publicação: 01/09/2015

\begin{abstract}
Resumo
Este estudo teve como objetivo caracterizar o estrato arbóreo das comunidades de cerrado sensu stricto mediante avaliações da riqueza, estrutura e diversidade. Os dados da vegetação foram obtidos

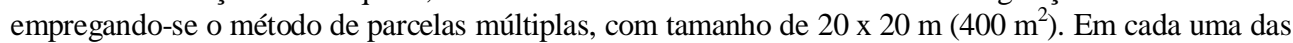
82 unidades amostrais foram obtidas a altura total e as circunferências de todas as plantas arbóreas com perímetro a $0,30 \mathrm{~m}$ do nível do solo (PAB) maior ou igual a $15,7 \mathrm{~cm}(\mathrm{DAB} \geq 5,0 \mathrm{~cm})$. A partir da parcela $60\left(24.000 \mathrm{~m}^{2}\right.$ da área amostrada) a curva estabilizou-se com a ocorrência de 106 espécies, distribuídas entre 81 gêneros e 36 famílias, indicando que a amostragem foi suficiente para caracterizar e avaliar as vegetações de cerrado sensu stricto estudadas. As espécies com maior VI foram: Qualea parviflora, Curatella americana, Davilla elliptica, Qualea grandiflora, Pterodon emarginatus, Lafoensia pacari, Diptychandra aurantiaca, Myrcia albo-tomentosa, Caryocar brasiliense, Byrsonima pachyphylla, Byrsonima coccolobifolia, Hymenaea stigonocarpa, Callisthene fasciculata, Luehea paniculata, Magonia pubescens, Terminalia argentea, Erythroxylum deciduum, Couepia grandiflora e Pouteria ramiflora. A diversidade da vegetação arbórea encontrada na área estudada foi de 4,033 nats/ind. pelo índice de Shannon e de 0,975 pelo de Simpson, indicando alta diversidade florística.

Palavras-chave: Estrutura fitossociológica; riqueza; diversidade.
\end{abstract}

\begin{abstract}
Phytosociology of the arboreal communities remainders of sensu stricto cerrado in Central Brazil. This study has a objective to characterize the cerrado sensu stricto communities' arboreal stratum by evaluations of the richness, structures and diversity. Data of vegetation were obtained by the method of multiple plots, with size of $20 \times 20 \mathrm{~m}\left(400 \mathrm{~m}^{2}\right)$. In each one of the 82 patternless units were obtained the total height and the circumferences of all the arboreal plants with perimeter to $0.30 \mathrm{~m}$ from the level of the soil (PAB) larger or equal to $15.7 \mathrm{~cm}(\mathrm{DAB} 5.0 \mathrm{~cm})$. From the plot 60 (24.000 $\mathrm{m}^{2}$ out of the area used as sample) the curve is stabilized with the occurrence of 106 species, distributed between 81 genera and 36 families, indicating that the sampling was enough to characterize and to evaluate the vegetations of cerrado sensu stricto studied. The species with larger VI were: Qualea parviflora, Curatella americana, Davilla elliptica, Qualea grandiflora, Pterodon emarginatus, Lafoensia pacari, Diptychandra aurantiaca, Myrcia albo-tomentosa, Caryocar brasiliense, Byrsonima pachyphylla, Byrsonima coccolobifolia, Hymenaea stigonocarpa, Callisthene fasciculata, Luehea paniculata, Magonia pubescens, Terminalia argentea, Erythroxylum deciduum, Couepia grandiflora and Pouteria ramiflora. The diversity from the arboreal vegetation found in the area being studied was of 4.033 nats/ind. considering the Shannon Index and of 0.975 considering the Simpson Index, representing a great floristic diversity.

Keywords: Phytosociological structures; richness; diversity.
\end{abstract}

\section{INTRODUÇÃO}

Os Cerrados pertencem a um dos mais importantes Biomas do Brasil. Esse Bioma, segundo Borlaug (2002), ocupa $21 \%$ do território nacional e é considerada a última fronteira agrícola do planeta. De acordo com Coutinho (1990), o Cerrado se estende por aproximadamente 1,8 milhões de quilômetros quadrados, entretanto, a sua extensão original, incluindo aquelas definidas como "áreas de contato" entre

FLORESTA, Curitiba, PR, v. 45, n. 4, p. 769 - 780, out. / dez. 2015.

Finger, Z.; Finger, F. A.

ISSN eletrônico 1982-4688 / ISSN impresso 0015-3826 
Cerrado e outros tipos fisionômicos, pode chegar a 2,2 milhões de quilômetros quadrados (INSTITUTO GRASILEIRO DE GEOGRAFIA E ESTATÍSTICA (IBGE), 1993; PEREIRA JR., 1992). Esse Bioma abrange o Planalto Central brasileiro, cobrindo grande parte dos estados de Mato Grosso, Mato Grosso do Sul, Tocantins, Goiás, Minas Gerais, Maranhão, Piauí, e partes dos estados de São Paulo, Bahia, Pará, Paraná e Sergipe. Formações savânicas podem ainda ocorrer em partes dos estados do Amazonas, Amapá, Rondônia e Roraima.

É importante salientar que a flora desse Bioma não é totalmente conhecida, tendo sido feitas algumas tentativas de compilação da sua composição florística. De acordo com Sano et al. (2008), construindo novas listas, adotando a estratégia de consultar prioritariamente publicações existentes, conseguiram compilar 12.356 espécies presentes espontaneamente nesse bioma, sendo que, deste total, 11.627 pertencem à flora vascular nativa. A riqueza florística apresentada por esses últimos autores ultrapassou em muito as compilações preexistentes, quase dobrando em número quando comparada à compilação de Mendonça et al. (1998). Essas estimativas sugerem grande riqueza florística no bioma, distribuída em uma grande variedade de paisagens e tipos fitofisionômicos, mostrando, ainda segundo esses mesmos autores, que o Cerrado é muito mais rico do que se supunha, e muitas de suas tipologias, com flora específica, são endêmicas da América do Sul e do Brasil.

Dessa forma, partindo-se da hipótese de que o conhecimento tanto dos componentes bióticos e abióticos da paisagem permite um melhor entendimento da dinâmica ambiental, este estudo procurou contribuir para o conhecimento da flora de comunidades arbóreas de cerrado na região de Chapada dos Guimarães e Baixada Cuiabana, tendo como objetivo específico caracterizar a riqueza florística, estrutura fitossociológica e diversidade do estrato arbóreo dessas áreas.

\section{MATERIAL E MÉTODOS}

O estudo foi desenvolvido no estado de Mato Grosso restringindo-se a áreas cobertas por vegetação com fisionomia savânica do tipo cerrado sensu stricto remanescentes, que compreendem, respectivamente, um alto platô - a Chapada dos Guimarães - e uma grande planície baixa - a Baixada Cuiabana. Os fragmentos estudados situam-se: na Chapada dos Guimarães entre as coordenadas geográficas $15^{\circ} 13^{\prime}$ e $15^{\circ} 15^{\prime}$ sul e $55^{\circ} 30^{\prime}$ e $55^{\circ} 33^{\prime}$ oeste, a uma altitude aproximada de 454 m s.n.m. no ponto mais alto e $365 \mathrm{~m}$ s.n.m. no ponto mais baixo; na Baixada Cuiabana situam-se entre as coordenadas geográficas $15^{\circ} 55^{\prime}$ e $15^{\circ} 58^{\prime}$ sul e $55^{\circ} 39^{\prime}$ e $55^{\circ} 42^{\prime}$ oeste, a uma altitude aproximada de $500 \mathrm{~m}$ s.n.m. no ponto mais alto e $155 \mathrm{~m}$ s.n.m. no ponto mais baixo. Evidências visuais e históricas sugerem que estas áreas foram submetidas há muitos anos à ação de fogo de baixa intensidade.

O clima, nessa região, de acordo com a classificação de Köppen, é do tipo Aw, isto é, tropical continental sempre quente, definido por duas estações: verão chuvoso, de outubro a abril, e inverno seco, de maio a setembro.

O tipo de vegetação na região é representado predominantemente por Savana, com suas diferentes variações fisionômicas. Na Chapada dos Guimarães, a Savana está representada basicamente pelos cerrados interfluviais, que se encontram entremeados por cerradão como transições para as florestas de galeria, que aparecem ao longo dos cursos d'água, e por campos, constituídos pelas veredas e pelos capões de buritis (Mauritia flexuosa L.f.), nelas predominando um brejo graminoso e, eventualmente, aparecendo os buritis nos locais de afloramento do lençol freático. Na Baixada Cuiabana, além dos cerrados interfluviais que representam o principal tipo fisionômico juntamente com os cerrados do tipo sensu stricto, mais ao sul, onde já começa a aparecer a vegetação que faz parte do Pantanal matogrossense, podem ser observadas as formações do tipo cerradão como transições para as florestas estacionais, decíduas e semidecíduas.

A área de estudos na região da Chapada dos Guimarães pertence a unidade litoestratigráfica Formação Furnas, do Grupo Paraná os solos, e de acordo com o Sistema Brasileiro de Classificação de Solos (EMPRESA BRASILEIRA DE PESQUISA AGROPECUÁRIA (EMBRAPA), 1999), nas partes mais baixas, os solos são classificados como Cambissolos Háplicos Tb Eutroférricos, e, nas partes mais altas, como Neossolos Quartzarênicos Órticos; já a área de estudos localizada na Baixada Cuiabana petence ao Grupo Cuiabá, sendo que os solos, nas partes mais baixas são classificados como Argissolos Vermelho Amarelo Eutrófico Típico e Distrófico Típico, e, nas partes mais altas são classificados como Cambissolos Háplicos Tb Distróficos lépticos.

Os dados da vegetação foram obtidos empregando-se o método de área fixa ou de parcelas múltiplas, como preconizado por Mueller-Dombois e Ellenberg (1974), com tamanho de 20 x $20 \mathrm{~m}$ 
$\left(400 \mathrm{~m}^{2}\right)$, dispostas aleatoriamente nas áreas de estudos. Foram instaladas aleatoriamente 82 parcelas, totalizando $32.800 \mathrm{~m}^{2}$ de área amostral.

Em cada uma das 82 unidades amostrais, com o auxílio de fita métrica, foram obtidas as circunferências de todas as plantas arbóreas com perímetro a 0,30 m do nível do solo (Perímetro na Altura da Base - PAB) maior ou igual a 15,7 cm (Diâmetro na Altura da Base - DAB $\geq 5,0 \mathrm{~cm}$ ). A altura total das plantas foi obtida com o uso de régua graduada ou de hipsômetro de Blume Leiss.

A identificação do material botânico foi realizada por padrões clássicos utilizados pela taxonomia, com base em caracteres morfológicos florais e vegetativos, com a utilização de coleções botânicas, pela comparação de exsicatas coletadas com material catalogado no Herbário Central da Universidade Federal de Mato Grosso, e também pela consulta à literatura e especialistas. As espécies foram organizadas de acordo com as famílias reconhecidas pelo Angiosperm Phylogeny Group III (APG II, 2009). Os nomes científicos foram conferidos com o Missouri Botanical Garden.

A suficiência de amostragem foi obtida com base na análise da curva do coletor construída por meio do estimador não paramétrico Jackknife, que se baseia na ocorrência de espécies e no número de parcelas para estimar o total de espécies na comunidade. Os cálculos foram realizados por meio dos programas Excel versão 9.0.0.2719 e STATISTICA versão 7 desenvolvidos pela empresa Microsoft Corporation, e EstimateS for Windows versão 8.2.0. (COLWELL, 2006)

Os parâmetros fitossociológicos foram calculados segundo Mueller-Dombois e Ellenberg (1974). O valor de importância (VI) e o valor de cobertura (VC) foram calculados segundo Kent e Coker (1992). A diversidade foi determinada por meio dos índices de diversidade de Shannon e de Simpson. Os cálculos foram realizados por meio do programa Excel, versão 9.0.0.2719, desenvolvido pela empresa Microsoft Corporation.

\section{RESULTADOS E DISCUSSÃO}

Por meio da construção da curva média de acumulação de espécies obteve-se uma estimativa de riqueza de 123,19 espécies para a área estudada, através do estimador não-paramétrico Jackknife 1, com a curva tendendo a assíntota com o esforço realizado a partir da parcela 60 (Figura 1).

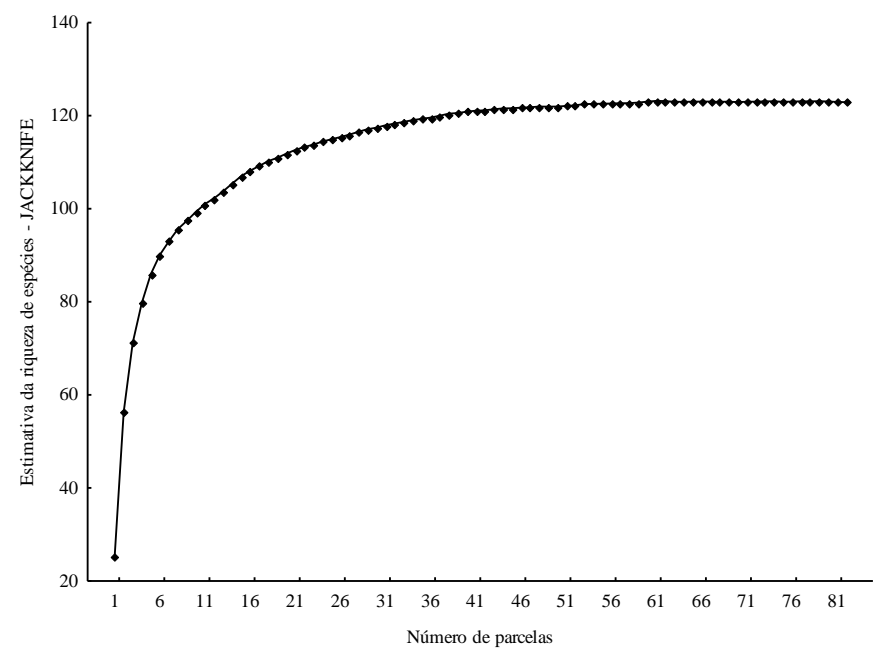

Figura 1. Curva de acumulação de espécies obtida através do estimador não paramétrico Jackknife em relação à área amostrada, em cerrado stricto sensu, Mato Grosso, Brasil.

Figure 1. Curves of accumulation of species obtained by the no parametric Jackknife estimator in relation to the sample area, in cerrado stricto sensu, Mato Grosso, Brazil.

\section{Caracterização geral das áreas estudadas}

A vegetação estudada apresenta áreas de contato com fitofisionomias do tipo cerradão, veredas, matas ciliares, matas de galeria e matas de galeria inundáveis, corroborado pela presença de Astronium fraxinifolium, Myracrodruon urundeuva, Tapirira guianensis, Protium heptaphyllum, Copaifera lagsdorffii, Emmotum nitens, Physocalimma scaberrimum, Miconia albicans, Anadenanthera peregrina var. falcata, Plathymenia reticulata, Siparuna guianensis, Dipteryx alata, Machaerium acutifolium, Plathypodium elegans, Zanthoxylum rigidum subsp. hasslerianum, Dilodendron bipinnatum e Guazuma

FLORESTA, Curitiba, PR, v. 45, n. 4, p. 769 - 780, out. / dez. 2015.

Finger, Z.; Finger, F. A.

ISSN eletrônico 1982-4688 / ISSN impresso 0015-3826 
ulmifolia (Tabela 1).

Foram encontradas, nas comunidades de cerrado sensu stricto estudadas em Chapada dos Guimarães e Baixada Cuiabana, Mato Grosso, Brasil, 114 espécies arbóreas entre indivíduos com DAB $\geq 5,0$.

A localização das parcelas na área de estudos, com grande variação em relevo, em altitude e nas características edáficas, certamente contribuiu para a elevada riqueza florística encontrada.

As famílias mais bem representadas foram Fabaceae, Myrtaceae, Vochysiaceae, Bignoniaceae, Malvaceae, Apocynaceae, Annonaceae, Malpighiaceae, Anacardiaceae, Rubiaceae, Chrysobalanaceae, Melastomataceae. Os resultados encontrados confirmam as informações de Gentry et al. (1997), Mendonça et al. (1998) e Felfili et al. (2002), que também encontraram em cerrados as Fabaceae, Myrtaceae e Vochysiaceae como as mais representativas.

Tabela 1. Parâmetros fitossociológicos das espécies do estrato arbóreo em comunidades de cerrado sensu stricto, nos municípios de Chapada dos Guimarães e Santo Antônio de Leverger, Mato Grosso, Brasil.

Table 1. Phytosociological parameters of the species of the arboreal stratum in communities of cerrado sensu stricto, in the municipal districts of Chapada dos Guimarães and Santo Antônio de Leverger, Mato Grosso, Brazil.

\begin{tabular}{|c|c|c|c|c|c|c|c|c|c|}
\hline Espécies & Família & $\begin{array}{l}\mathbf{F A}_{\mathbf{i}} \\
(\%)\end{array}$ & $\begin{array}{l}\mathbf{F R}_{\mathbf{i}} \\
(\%)\end{array}$ & $\begin{array}{c}\mathbf{D A}_{\mathbf{i}} \\
\text { ind/ha }\end{array}$ & $\begin{array}{l}\mathbf{D R}_{\mathbf{i}} \\
(\%)\end{array}$ & $\begin{array}{l}\mathrm{DoA}_{\mathbf{i}} \\
\mathrm{m}^{2} / \mathrm{ha}\end{array}$ & $\begin{array}{l}\operatorname{DoR}_{\mathbf{i}} \\
(\%)\end{array}$ & V I & V C \\
\hline Qualea parviflora Mart. & VOCHYSIACEAE & 90,24 & 3,57 & 115,24 & 6,62 & 1,24 & 6,32 & 16,52 & 12,95 \\
\hline Curatella americana $\mathrm{L}$. & DILLENIACEAE & 62,20 & 2,46 & 93,90 & 5,40 & 1,30 & 6,63 & 14,49 & 12,03 \\
\hline Pterodon emarginatus Vog. & FABACEAE & 43,90 & 1,74 & 22,87 & 1,31 & 1,29 & 6,58 & 9,63 & 7,89 \\
\hline Qualea grandiflora Mart. & VOCHYSIACEAE & 71,95 & 2,85 & 67,07 & 3,86 & 0,77 & 3,90 & 10,60 & 7,76 \\
\hline Davilla elliptica A. St. Hill. & DILLENIACEAE & 84,15 & 3,33 & 90,24 & 5,19 & 0,42 & 2,15 & 10,67 & 7,34 \\
\hline Lafoensia pacari A. St. Hil. & LYTHRACEAE & 56,10 & 2,22 & 71,95 & 4,14 & 0,58 & 2,96 & 9,32 & 7,10 \\
\hline Diptychandra aurantiaca Tul. & FABACEAE & 59,76 & 2,36 & 51,83 & 2,98 & 0,74 & 3,80 & 9,14 & 6,78 \\
\hline Caryocar brasiliense Cambess. & CARYOCARACEAE & 40,24 & 1,59 & 35,67 & 2,05 & 0,87 & 4,46 & 8,10 & 6,51 \\
\hline Callisthene fasciculata (Spr.) Mart. & FABACEAE & 6,10 & 0,24 & 53,35 & 3,07 & 0,63 & 3,19 & 6,50 & 6,26 \\
\hline Myrcia albotomentosa DC. & MYRTACEAE & 57,32 & 2,27 & 75,00 & 4,31 & 0,34 & 1,74 & 8,32 & 6,06 \\
\hline Byrsonima pachyphylla Juss. & MALPIGHIACEAE & 39,02 & 1,54 & 49,70 & 2,86 & 0,48 & 2,46 & 6,86 & 5,31 \\
\hline $\begin{array}{l}\text { Hymenaea stigonocarpa Mart. ex } \\
\text { Hayne }\end{array}$ & FABACEAE & 43,90 & 1,74 & 26,83 & 1,54 & 0,64 & 3,26 & 6,54 & 4,80 \\
\hline Byrsonima coccolobifolia Kunth & MALPIGHIACEAE & 57,32 & 2,27 & 37,50 & 2,16 & 0,44 & 2,23 & 6,65 & 4,38 \\
\hline Luehea paniculata Mart. & MALVACEAE & 37,80 & 1,50 & 34,45 & 1,98 & 0,43 & 2,20 & 5,68 & 4,18 \\
\hline Magonia pubescens A. St. Hil. & SAPINDACEAE & 40,24 & 1,59 & 39,02 & 2,24 & 0,35 & 1,80 & 5,63 & 4,04 \\
\hline Terminalia argentea Mart. & COMBRETACEAE & 46,34 & 1,83 & 35,37 & 2,03 & 0,32 & 1,63 & 5,50 & 3,66 \\
\hline Xylopia aromatica (Lam.) Mart. & ANNONACEAE & 31,71 & 1,25 & 35,98 & 2,07 & 0,30 & 1,54 & 4,86 & 3,61 \\
\hline $\begin{array}{l}\text { Couepia grandiflora (Mart. et } \\
\text { Zucc.) Benth. ex Hook. }\end{array}$ & CHRYSOBALANACEAE & 37,80 & 1,50 & 24,39 & 1,40 & 0,42 & 2,12 & 5,02 & 3,53 \\
\hline Tachigali aurea Tul. & FABACEAE & 34,15 & 1,35 & 26,52 & 1,52 & 0,37 & 1,90 & 4,78 & 3,43 \\
\hline Erythroxylum deciduum St. Hil. & ERYTHROXYLACEAE & 47,56 & 1,88 & 40,55 & 2,33 & 0,19 & 0,99 & 5,20 & 3,32 \\
\hline $\begin{array}{l}\text { Pouteria ramiflora (Mart.) } \\
\text { Radlk. }\end{array}$ & SAPOTACEAE & 51,22 & 2,03 & 22,87 & 1,31 & 0,35 & 1,81 & 5,15 & 3,12 \\
\hline Dimorphandra mollis Benth. & FABACEAE & 51,22 & 2,03 & 22,87 & 1,31 & 0,28 & 1,42 & 4,76 & 2,73 \\
\hline $\begin{array}{l}\text { Vatairea macrocarpa (Benth.) } \\
\text { Ducke }\end{array}$ & FABACEAE & 41,46 & 1,64 & 23,48 & 1,35 & 0,27 & 1,36 & 4,35 & 2,71 \\
\hline Kielmeyera rubriflora Camb. & CLUSIACEAE & 30,49 & 1,21 & 22,87 & 1,31 & 0,24 & 1,22 & 3,74 & 2,54 \\
\hline $\begin{array}{l}\text { Ouratea hexasperma (St. Hil.) } \\
\text { Baill. }\end{array}$ & OCHNACEAE & 50,00 & 1,98 & 29,88 & 1,72 & 0,16 & 0,80 & 4,50 & 2,52 \\
\hline $\begin{array}{l}\text { Handroanthus ochraceus } \\
\text { (Cham.) Mattos }\end{array}$ & BIGNONIACEAE & 42,68 & 1,69 & 23,48 & 1,35 & 0,23 & 1,15 & 4,19 & 2,50 \\
\hline Roupala montana Aubl. & PROTEACEAE & 45,12 & 1,79 & 27,44 & 1,58 & 0,18 & 0,92 & 4,28 & 2,50 \\
\hline Handroanthus aureus Mattos & BIGNONIACEAE & 45,12 & 1,79 & 14,02 & 0,81 & 0,33 & 1,67 & 4,26 & 2,47 \\
\hline $\begin{array}{l}\text { Mezilaurus wanderwerffii F. M. } \\
\text { Alves \& J. b. Baitello }\end{array}$ & LAURACEAE & 24,39 & 0,97 & 18,90 & 1,09 & 0,22 & 1,12 & 3,17 & 2,20 \\
\hline $\begin{array}{l}\text { Connarus suberosus. var. fulvus } \\
\text { (Planch.) Forero }\end{array}$ & CONNARACEAE & 62,20 & 2,46 & 26,22 & 1,51 & 0,13 & 0,64 & 4,61 & 2,15 \\
\hline Vochysia cinnamomea Pohl & VOCHYSIACEAE & 35,37 & 1,40 & 20,12 & 1,16 & 0,19 & 0,97 & 3,53 & 2,13 \\
\hline
\end{tabular}

FLORESTA, Curitiba, PR, v. 45, n. 4, p. 769 - 780, out. / dez. 2015. Finger, Z.; Finger, F. A 


\begin{tabular}{|c|c|c|c|c|c|c|c|c|c|}
\hline $\begin{array}{l}\text { Eriotheca gracilipes (K. Schum.) } \\
\text { A. Robyns }\end{array}$ & MALVACEAE & 36,59 & 1,45 & 16,77 & 0,96 & 0,23 & 1,16 & 3,57 & 2,12 \\
\hline $\begin{array}{l}\text { Anadenanthera peregrina var. } \\
\text { falcata (Benth.) Altschul }\end{array}$ & FABACEAE & 26,83 & 1,06 & 13,11 & 0,75 & 0,24 & 1,25 & 3,06 & 2,00 \\
\hline Mouriri pusa Gardn. & MELASTOMATACEAE & 28,05 & 1,11 & 18,29 & 1,05 & 0,18 & 0,92 & 3,08 & 1,97 \\
\hline $\begin{array}{l}\text { Pseudobombax tomentosum } \\
\text { (Mart. \& Zucc.) A. Robyns }\end{array}$ & MALVACEAE & 25,61 & 1,01 & 12,50 & 0,72 & 0,24 & 1,24 & 2,98 & 1,96 \\
\hline Aspidosperma tomentosum Mart. & APOCYNACEAE & 34,15 & 1,35 & 13,41 & 0,77 & 0,23 & 1,18 & 3,31 & 1,95 \\
\hline $\begin{array}{l}\text { Salvertia convallariaeodora } \text { St. } \\
\text { Hil. }\end{array}$ & VOCHYSIACEAE & 32,93 & 1,30 & 13,11 & 0,75 & 0,24 & 1,20 & 3,26 & 1,95 \\
\hline Andira cujabensis Benth. & FABACEAE & 37,80 & 1,50 & 12,80 & 0,74 & 0,22 & 1,12 & 3,35 & 1,85 \\
\hline Qualea multiflora Mart. & VOCHYSIACEAE & 39,02 & 1,54 & 21,04 & 1,21 & 0,11 & 0,57 & 3,33 & 1,78 \\
\hline Mouriri elliptica Mart. & MELASTOMATACEAE & 24,39 & 0,97 & 18,60 & 1,07 & 0,13 & 0,69 & 2,72 & 1,75 \\
\hline $\begin{array}{l}\text { Licania sclerophylla (Hook. f.) } \\
\text { Fritsch }\end{array}$ & CHRYSOBALANACEAE & 28,05 & 1,11 & 14,02 & 0,81 & 0,18 & 0,91 & 2,83 & 1,72 \\
\hline $\begin{array}{l}\text { Byrsonima verbascifolia (L.) } \\
\text { Rich. ex Juss. }\end{array}$ & MALPIGHIACEAE & 41,46 & 1,64 & 18,90 & 1,09 & 0,12 & 0,59 & 3,32 & 1,68 \\
\hline Annona crassiflora Mart. & ANNONACEAE & 36,59 & 1,45 & 12,50 & 0,72 & 0,16 & 0,79 & 2,96 & 1,51 \\
\hline Bowdichia virgilioides Kunth & FABACEAE & 15,85 & 0,63 & 6,71 & 0,39 & 0,21 & 1,08 & 2,09 & 1,47 \\
\hline Vochysia rufa Mart. & VOCHYSIACEAE & 24,39 & 0,97 & 14,33 & 0,82 & 0,12 & 0,64 & 2,43 & 1,46 \\
\hline $\begin{array}{l}\text { Kielmeyera coriacea Mart. \& } \\
\text { Zucc. }\end{array}$ & CLUSIACEAE & 37,80 & 1,50 & 14,33 & 0,82 & 0,12 & 0,62 & 2,94 & 1,44 \\
\hline Plathymenia reticulata Benth. & FABACEAE & 24,39 & 0,97 & 10,98 & 0,63 & 0,13 & 0,68 & 2,28 & 1,31 \\
\hline Diospyrus hispida A. DC. & EBENACEAE & 42,68 & 1,69 & 14,02 & 0,81 & 0,10 & 0,50 & 3,00 & 1,31 \\
\hline Astronium fraxinifolium Schott & ANACARDIACEAE & 24,39 & 0,97 & 13,41 & 0,77 & 0,10 & 0,52 & 2,26 & 1,30 \\
\hline Eugenia bimarginata DC. & MYRTACEAE & 26,83 & 1,06 & 13,41 & 0,77 & 0,07 & 0,34 & 2,17 & 1,11 \\
\hline $\begin{array}{l}\text { Protium heptaphyllum (Aubl.) } \\
\text { March. }\end{array}$ & BURSERACEAE & 17,07 & 0,68 & 10,67 & 0,61 & 0,09 & 0,44 & 1,73 & 1,05 \\
\hline $\begin{array}{l}\text { Myracrodruon urundeuva } \\
\text { Allemão }\end{array}$ & ANACARDIACEAE & 15,85 & 0,63 & 9,45 & 0,54 & 0,09 & 0,47 & 1,64 & 1,02 \\
\hline Buchenavia tomentosa Eichler & COMBRETACEAE & 15,85 & 0,63 & 5,49 & 0,32 & 0,13 & 0,67 & 1,61 & 0,98 \\
\hline Myrtaceae 8 & MYRTACEAE & 18,29 & 0,72 & 9,45 & 0,54 & 0,07 & 0,37 & 1,64 & 0,91 \\
\hline $\begin{array}{l}\text { Aspidosperma macrocarpon } \\
\text { Mart. }\end{array}$ & APOCYNACEAE & 14,63 & 0,58 & 3,96 & 0,23 & 0,12 & 0,62 & 1,43 & 0,85 \\
\hline Eugenia dysenterica DC. & MYRTACEAE & 15,85 & 0,63 & 7,01 & 0,40 & 0,08 & 0,42 & 1,45 & 0,83 \\
\hline $\begin{array}{l}\text { Guettarda viburnoides Cham. \& } \\
\text { Schltdl. }\end{array}$ & RUBIACEAE & 12,20 & 0,48 & 7,93 & 0,46 & 0,04 & 0,22 & 1,16 & 0,68 \\
\hline $\begin{array}{l}\text { Stryphnodendron adstringens } \\
\text { (Mart.) Coville }\end{array}$ & FABACEAE & 14,63 & 0,58 & 6,71 & 0,39 & 0,06 & 0,29 & 1,26 & 0,68 \\
\hline Dipteryx alata Vogel & FABACEAE & 6,10 & 0,24 & 3,05 & 0,18 & 0,10 & 0,49 & 0,91 & 0,67 \\
\hline Myrtaceae 1 & MYRTACEAE & 19,51 & 0,77 & 6,40 & 0,37 & 0,05 & 0,26 & 1,40 & 0,63 \\
\hline Rhamnidium elaeocarpum Reiss. & RHAMNACEAE & 15,85 & 0,63 & 7,01 & 0,40 & 0,03 & 0,16 & 1,19 & 0,57 \\
\hline $\begin{array}{l}\text { Bowdichia major (Mart.) Mart. } \\
\text { ex Benth. }\end{array}$ & FABACEAE & 12,20 & 0,48 & 3,05 & 0,18 & 0,07 & 0,38 & 1,04 & 0,56 \\
\hline Coccoloba mollis Casar. & POLYGONACEAE & 3,66 & 0,14 & 4,57 & 0,26 & 0,05 & 0,28 & 0,68 & 0,54 \\
\hline Rourea induta Planch. & CONNARACEAE & 20,73 & 0,82 & 7,32 & 0,42 & 0,02 & 0,12 & 1,36 & 0,54 \\
\hline Tapirira guianensis Aubl. & ANACARDIACEAE & 9,76 & 0,39 & 6,10 & 0,35 & 0,04 & 0,19 & 0,92 & 0,54 \\
\hline Erythroxylum suberosum A. St. Hil. & ERYTROXYLACEAE & 12,20 & 0,48 & 6,71 & 0,39 & 0,03 & 0,13 & 1,00 & 0,52 \\
\hline Emmotum nitens (Benth.) Miers & ICACINACEAE & 4,88 & 0,19 & 1,83 & 0,11 & 0,07 & 0,38 & 0,68 & 0,48 \\
\hline Strychnos pseudoquina A. St. Hil. & LOGANIACEAE & 13,41 & 0,53 & 4,88 & 0,28 & 0,03 & 0,17 & 0,98 & 0,45 \\
\hline $\begin{array}{l}\text { Himatanthus obovatus (M. Arg.) } \\
\text { Wood. }\end{array}$ & APOCYNACEAE & 17,07 & 0,68 & 5,79 & 0,33 & 0,02 & 0,10 & 1,11 & 0,44 \\
\hline Annona coriacea Mart. & ANNONACEAE & 13,41 & 0,53 & 4,57 & 0,26 & 0,03 & 0,17 & 0,96 & 0,43 \\
\hline $\begin{array}{l}\text { Peltogyne confertiflora (Mart. ex } \\
\text { Hayne) Benth. }\end{array}$ & FABACEAE & 8,54 & 0,34 & 2,74 & 0,16 & 0,04 & 0,22 & 0,72 & 0,38 \\
\hline Simarouba versicolor A. St. Hil. & SIMAROUBACEAE & 14,63 & 0,58 & 3,96 & 0,23 & 0,03 & 0,15 & 0,95 & 0,38 \\
\hline Ecclinusa ramiflora Mart. & SAPOTACEAE & 9,76 & 0,39 & 4,57 & 0,26 & 0,02 & 0,11 & 0,75 & 0,37 \\
\hline $\begin{array}{l}\text { Pseudobombax longiflorum } \\
\text { (Mart. \& Zucc.) A. Robyns }\end{array}$ & MALVACEAE & 2,44 & 0,10 & 0,61 & 0,04 & 0,06 & 0,32 & 0,46 & 0,36 \\
\hline Brosimum gaudichaudii Trec. & MORACEAE & 10,98 & 0,43 & 4,27 & 0,25 & 0,02 & 0,11 & 0,79 & 0,36 \\
\hline Myrtaceae 11 & MYRTACEAE & 3,66 & 0,14 & 3,66 & 0,21 & 0,02 & 0,10 & 0,46 & 0,31 \\
\hline $\begin{array}{l}\text { Aspidosperma spruceanum } \\
\text { Benth. ex M. Arg. }\end{array}$ & APOCYNACEAE & 10,98 & 0,43 & 3,05 & 0,18 & 0,03 & 0,13 & 0,74 & 0,31 \\
\hline
\end{tabular}

FLORESTA, Curitiba, PR, v. 45, n. 4, p. 769 - 780, out. / dez. 2015.

Finger, Z.; Finger, F. A.

ISSN eletrônico 1982-4688 / ISSN impresso 0015-3826

DOI: $10.5380 /$ rf.v45i4.30860 


\begin{tabular}{|c|c|c|c|c|c|c|c|c|c|}
\hline Leguminosae 1 & FABACEAE & 6,10 & 0,24 & 3,05 & 0,18 & 0,02 & 0,12 & 0,54 & 0,29 \\
\hline $\begin{array}{l}\text { Rudgea viburnoides (Cham.) } \\
\text { Benth. }\end{array}$ & RUBIACEAE & 4,88 & 0,19 & 3,05 & 0,18 & 0,02 & 0,10 & 0,47 & 0,28 \\
\hline $\begin{array}{l}\text { Hancornia speciosa var. } \\
\text { cuyabensis Malme }\end{array}$ & APOCYNACEAE & 12,20 & 0,48 & 3,05 & 0,18 & 0,02 & 0,10 & 0,76 & 0,28 \\
\hline Copaifera langsdorffii Desf. & FABACEAE & 6,10 & 0,24 & 2,74 & 0,16 & 0,01 & 0,07 & 0,47 & 0,23 \\
\hline $\begin{array}{l}\text { Mezilaurus crassiramea (Meisn.) } \\
\text { Taub. ex Mez }\end{array}$ & LAURACEAE & 4,88 & 0,19 & 1,52 & 0,09 & 0,03 & 0,13 & 0,41 & 0,22 \\
\hline $\begin{array}{l}\text { Tocoyena formosa (Cham. \& } \\
\text { Schltdl.) K. Sch. }\end{array}$ & RUBIACEAE & 8,54 & 0,34 & 2,74 & 0,16 & 0,01 & 0,06 & 0,55 & 0,21 \\
\hline $\begin{array}{l}\text { Peritassa campestris (Camb.) A. } \\
\text { C. Sm. }\end{array}$ & HYPOCRATEACEAE & 7,32 & 0,29 & 1,83 & 0,11 & 0,02 & 0,10 & 0,49 & 0,20 \\
\hline Myrtaceae 5 & MYRTACEAE & 4,88 & 0,19 & 1,83 & 0,11 & 0,02 & 0,09 & 0,39 & 0,20 \\
\hline Machaerium acutifolium Vog. & FABACEAE & 8,54 & 0,34 & 2,13 & 0,12 & 0,01 & 0,07 & 0,53 & 0,19 \\
\hline Agonandra brasiliensis Miers & OPILIACEAE & 6,10 & 0,24 & 1,83 & 0,11 & 0,01 & 0,05 & 0,40 & 0,16 \\
\hline Andira anthelmia (Vell.) Macbr. & FABACEAE & 4,88 & 0,19 & 2,13 & 0,12 & 0,01 & 0,03 & 0,34 & 0,15 \\
\hline Licania $s p$ & CHRYSOBALANACEAE & 4,88 & 0,19 & 1,52 & 0,09 & 0,01 & 0,05 & 0,33 & 0,14 \\
\hline Jacaranda cuspidifolia Mart. & BIGNONIACEAE & 4,88 & 0,19 & 1,22 & 0,07 & 0,01 & 0,06 & 0,32 & 0,13 \\
\hline Myrtaceae 7 & MYRTACEAE & 3,66 & 0,14 & 0,91 & 0,05 & 0,01 & 0,05 & 0,25 & 0,11 \\
\hline $\begin{array}{l}\text { Physocalymma scaberrimum } \\
\text { Pohl }\end{array}$ & LYTHRACEAE & 2,44 & 0,10 & 0,61 & 0,04 & 0,01 & 0,07 & 0,20 & 0,10 \\
\hline Myrtaceae 9 & MYRTACEAE & 3,66 & 0,14 & 1,22 & 0,07 & 0,01 & 0,03 & 0,24 & 0,10 \\
\hline $\begin{array}{l}\text { Dilodendron bipinnatum Radlk. } \\
\text { Handroanthus vellosoi (Toledo) }\end{array}$ & SAPINDACEAE & 3,66 & 0,14 & 1,22 & 0,07 & 0,00 & 0,02 & 0,24 & 0,09 \\
\hline Mattos & BIGNONIACEAE & 2,44 & 0,10 & 0,61 & 0,04 & 0,01 & 0,06 & 0,19 & 0,09 \\
\hline Miconia albicans (Sw.) Tr. & MELASTOMATACEAE & 3,66 & 0,14 & 0,91 & 0,05 & 0,01 & 0,03 & 0,23 & 0,08 \\
\hline Leguminosae 2 & FABACEAE & 3,66 & 0,14 & 0,91 & 0,05 & 0,01 & 0,03 & 0,22 & 0,08 \\
\hline Myrtaceae 4 & MYRTACEAE & 2,44 & 0,10 & 0,91 & 0,05 & 0,00 & 0,02 & 0,17 & 0,08 \\
\hline Leptolobium elegans Vogel & FABACEAE & 2,44 & 0,10 & 0,61 & 0,04 & 0,01 & 0,04 & 0,17 & 0,07 \\
\hline $\begin{array}{l}\text { Handroanthus roseo-albus } \\
\text { (Ridl.) Mattos }\end{array}$ & BIGNONIACEAE & 2,44 & 0,10 & 0,61 & 0,04 & 0,01 & 0,04 & 0,17 & 0,07 \\
\hline $\begin{array}{l}\text { Zanthoxylum rigidum subsp. } \\
\text { hasslerianum (Chodat) Reynel }\end{array}$ & RUTACEAE & 2,44 & 0,10 & 0,61 & 0,04 & 0,00 & 0,02 & 0,15 & 0,06 \\
\hline Platypodium elegans Vog. & FABACEAE & 2,44 & 0,10 & 0,61 & 0,04 & 0,00 & 0,02 & 0,15 & 0,05 \\
\hline Myrtaceae 10 & MYRTACEAE & 1,22 & 0,05 & 0,30 & 0,02 & 0,01 & 0,03 & 0,10 & 0,05 \\
\hline Guazuma ulmifolia Lam. & MALVACEAE & 2,44 & 0,10 & 0,61 & 0,04 & 0,00 & 0,01 & 0,14 & 0,05 \\
\hline Myrtaceae 2 & MYRTACEAE & 2,44 & 0,10 & 0,61 & 0,04 & 0,00 & 0,01 & 0,14 & 0,04 \\
\hline Myrtaceae 3 & MYRTACEAE & 2,44 & 0,10 & 0,61 & 0,04 & 0,00 & 0,01 & 0,14 & 0,04 \\
\hline Myrtaceae 6 & MYRTACEAE & 1,22 & 0,05 & 0,30 & 0,02 & 0,00 & 0,02 & 0,08 & 0,04 \\
\hline Siparuna guianensis Aubl. & SIPARUNACEAE & 1,22 & 0,05 & 0,30 & 0,02 & 0,00 & 0,01 & 0,07 & 0,03 \\
\hline $\begin{array}{l}\text { Cardiopetalum calophyllum } \\
\text { Schltdl. }\end{array}$ & ANNONACEAE & 1,22 & 0,05 & 0,30 & 0,02 & 0,00 & 0,01 & 0,07 & 0,02 \\
\hline Cenostigma macrophyllum Tul. & FABACEAE & 1,22 & 0,05 & 0,30 & 0,02 & 0,00 & 0,00 & 0,07 & 0,02 \\
\hline Byrsonima basiloba A. Juss. & MALPIGHIACEAE & 1,22 & 0,05 & 0,30 & 0,02 & 0,00 & 0,00 & 0,07 & 0,02 \\
\hline Zeyheria montana Mart. & BIGNONIACEAE & 1,22 & 0,05 & 0,30 & 0,02 & 0,00 & 0,00 & 0,07 & 0,02 \\
\hline $\begin{array}{l}\text { Tachigali subvelutina (Benth.) } \\
\text { Oliveira-Filho }\end{array}$ & FABACEAE & 1,22 & 0,05 & 0,30 & 0,02 & 0,00 & 0,00 & 0,07 & 0,02 \\
\hline Bauhinia sp. & FABACEAE & 1,22 & 0,05 & 0,30 & 0,02 & 0,00 & 0,00 & 0,07 & 0,02 \\
\hline TOTAL & & 2526,83 & 100,00 & 1739,63 & 100,00 & 19,62 & 100,00 & 300,00 & 200,00 \\
\hline
\end{tabular}

FA $A_{\mathrm{i}}$ : frequência absoluta; $\mathrm{FR}_{\mathrm{i}}$ : frequência relativa; $\mathrm{DA}_{\mathrm{i}}$ : densidade absoluta; $\mathrm{DR}_{\mathrm{i}}$ : densidade relativa; DoA $\mathrm{A}_{\mathrm{i}}$ : dominância absoluta; DoRi: dominância relativa; VI: valor de importância; VC: valor de cobertura; ind/ha: indivíduos por hectare.

\section{Estrutura fitossociológica}

As estimativas de densidade e de área basal, por hectare, encontradas (Tabela 1) considerando os indivíduos arbóreos com $\mathrm{DAB} \geq 5,0 \mathrm{~cm}$, foram de 1.740 indivíduos e $19,62 \mathrm{~m}^{2}$, respectivamente.

As espécies com maior número de indivíduos por hectare foram: Qualea parviflora, Curatella americana, Davilla elliptica, Myrcia albo-tomentosa, Lafoensia pacari, Qualea grandiflora, Callisthene fasciculata, Diptychandra aurantiaca, Byrsonima pachyphylla, Erythroxylum deciduum, Magonia pubescens, Byrsonima coccolobifolia, Xylopia aromatica, Caryocar brasiliense, Terminalia argentea $\mathrm{e}$ 
Luehea paniculata (Tabela 1). Essas espécies representaram 14,04\% do total amostradas, entretanto, detiveram 53,28\% do total de indivíduos por hectare.

Em relação à área basal por hectare, as espécies que mais se destacaram foram: Curatella americana, Pterodon emarginatus, Qualea parviflora, Caryocar brasiliense, Qualea grandiflora, Diptychandra aurantiaca, Hymenaea stigonocarpa, Callisthene fasciculata, Lafoensia pacari, Byrsonima pachyphylla, Byrsonima coccolobifolia, Luehea paniculata, Davilla elliptica, Couepia grandiflora, Taghigali aurea e Pouteria ramiflora (Tabela 1). Essas espécies representaram apenas 14,04\% do total amostradas e, no entanto, detiveram juntas 55,97\% da área basal por ha.

As espécies com maior VI foram: Qualea parviflora, Curatella americana, Davilla elliptica, Qualea grandiflora, Pterodon emarginatus, Lafoensia pacari, Diptychandra aurantiaca, Myrcia albotomentosa, Caryocar brasiliense, Byrsonima pachyphylla, Byrsonima coccolobifolia, Hymenaea stigonocarpa, Callisthene fasciculata, Luehea paniculata, Magonia pubescens, Terminalia argentea, Erythroxylum deciduum, Couepia grandiflora e Pouteria ramiflora (Tabela 1). Essas espécies representaram $16,67 \%$ do total amostradas, entretanto, juntas, detiveram $56,78 \%$ do número total de indivíduos por ha, 60,24\% da área basal por ha e 51,84\% do VI.

As espécies com maior VC foram: Qualea parviflora, Curatella americana, Pterodon emarginatus, Qualea grandiflora, Davilla elliptica, Lafoensia pacari, Diptychandra aurantiaca, Caryocar brasiliense, Callisthene fasciculata, Myrcia albo-tomentosa, Byrsonima pachyphylla, Hymenaea stigonocarpa, Byrsonima coccolobifolia, Luehea paniculata, Magonia pubescens, Terminalia argentea, Xylopia aromatica, Couepia grandiflora e Tachigali aurea (Tabela 1). Estas espécies representaram apenas $16,67 \%$ do total amostrado, mas, juntas, detiveram $56,73 \%$ do número total de indivíduos por ha, $60,89 \%$ da área basal por ha e $58,80 \%$ do $V C$.

As espécies, com maior densidade e área basal por hectare e com maior $V I$ e $V C$, apresentam ampla distribuição nas regiões de cerrados em Mato Grosso. Furley et al. (1988) e Marimon et al. (1998), estudando cerrados aproximadamente $400 \mathrm{~km}$ a leste dos locais deste estudo, encontraram também, dentre as espécies com maior VI, Qualea parviflora, Curatella americana, Davilla elliptica, Lafoensia pacari e Callisthene fasciculata. Ratter et al. (1996), analisando a composição florística de 98 áreas nos cerrados do Brasil Central e em savanas amazônicas, verificaram a presença de Qualea parviflora, Curatella americana, respectivamente, em $60 \%$ e $71 \%$ das áreas. Essa tendência de um grupo pequeno de espécies prevalecer sobre as demais também se confirmou em outros estudos em áreas de cerrado sensu stricto no Brasil central (FELFILI; SILVA JÚNIOR, 1993; FELFILI et al., 1994; FELFILI, 1997; ROSSI et al., 1998; PIRES et al., 1999; FELFILI et al., 2002).

A curva de distribuição dos diâmetros apresentou a forma de um “j” invertido (Figura 2), ajustando-se ao mesmo padrão observado por Marimon Junior e Haridasan (2005) em cerrado sensu stricto e cerradão. Segundo Felfili e Silva Junior (1988), esse padrão é indicativo de fitofisionomias autoregenerativas.

O diâmetro médio dos indivíduos amostrados foi estimado em $10,47 \mathrm{~cm}$, com um coeficiente de variação dos diâmetros de todos indivíduos amostrados de 55,58\%. O maior número de indivíduos ocorreu entre 5,00 e 15,00 cm de diâmetro, que equivale a $83,16 \%$ do total de indivíduos amostrados. Cerca de 7,89\% das espécies encontram-se na classe 5 (C5) de diâmetro (15,00 a 13,01 cm); 16,67\% das espécies na classe 6 (C6) de diâmetro (13,00 a 11,01 cm); 24,56\% na classe 7 (C7) de diâmetro (11,00 a $9,01 \mathrm{~cm}) ; 28,07 \%$ na classe $8(\mathrm{C} 8)$ de diâmetro $(9,00$ a 7,01 cm); 13,16\% na classe 9 (C9) de diâmetro $(7,00$ a $5,00 \mathrm{~cm})$, sendo que apenas uma espécie apareceu na classe $1(\mathrm{C} 1)$ de diâmetro 35,00 a $27,01 \mathrm{~cm})$, uma na classe 2 (C2) de diâmetro $(27,00$ a 19,01 cm), duas na classe 3 (C3) de diâmetro $(19,00$ a $17,01 \mathrm{~cm})$ e sete na classe $4(\mathrm{C} 4)$ de diâmetro $(17,00$ a 15,01 cm).

Das espécies com maior VI, Pterodon emarginatus pertence à classe C2 de diâmetro; Caryocar brasiliense pertence à classe $\mathrm{C} 4$; Hymenaea stigonocarpa e Couepia grandiflora pertencem à classe C5; Curatella americana, Pouteria ramiflora, Diptychandra aurantiaca, Luehea paniculata, Byrsonima coccolobifolia e Callisthene fasciculata pertencem à classe C6; Qualea grandiflora, Byrsonima pachyphylla, Qualea parviflora, Terminalia argentea, Magonia pubescens, Lafoensia pacari pertencem à classe C7; Davilla elliptica, Erythroxylum deciduum e Myrcia albo-tomentosa pertencem a classe C8, e 90,35\% das espécies amostradas situaram-se nas classes 3, 4 e 5 .

A distribuição das alturas foi quase unimodal (Figura 3), ajustando-se aos mesmos padrões observados por Marimon Junior e Haridasan (2005) para o cerrado sensu stricto e para áreas bem conservadas de cerradão. Padrão semelhante foi observado por Felfili (1997) para uma comunidade arbórea em uma floresta de galeria no Brasil central.

FLORESTA, Curitiba, PR, v. 45, n. 4, p. 769 - 780, out. / dez. 2015.

Finger, Z.; Finger, F. A.

ISSN eletrônico 1982-4688 / ISSN impresso 0015-3826 


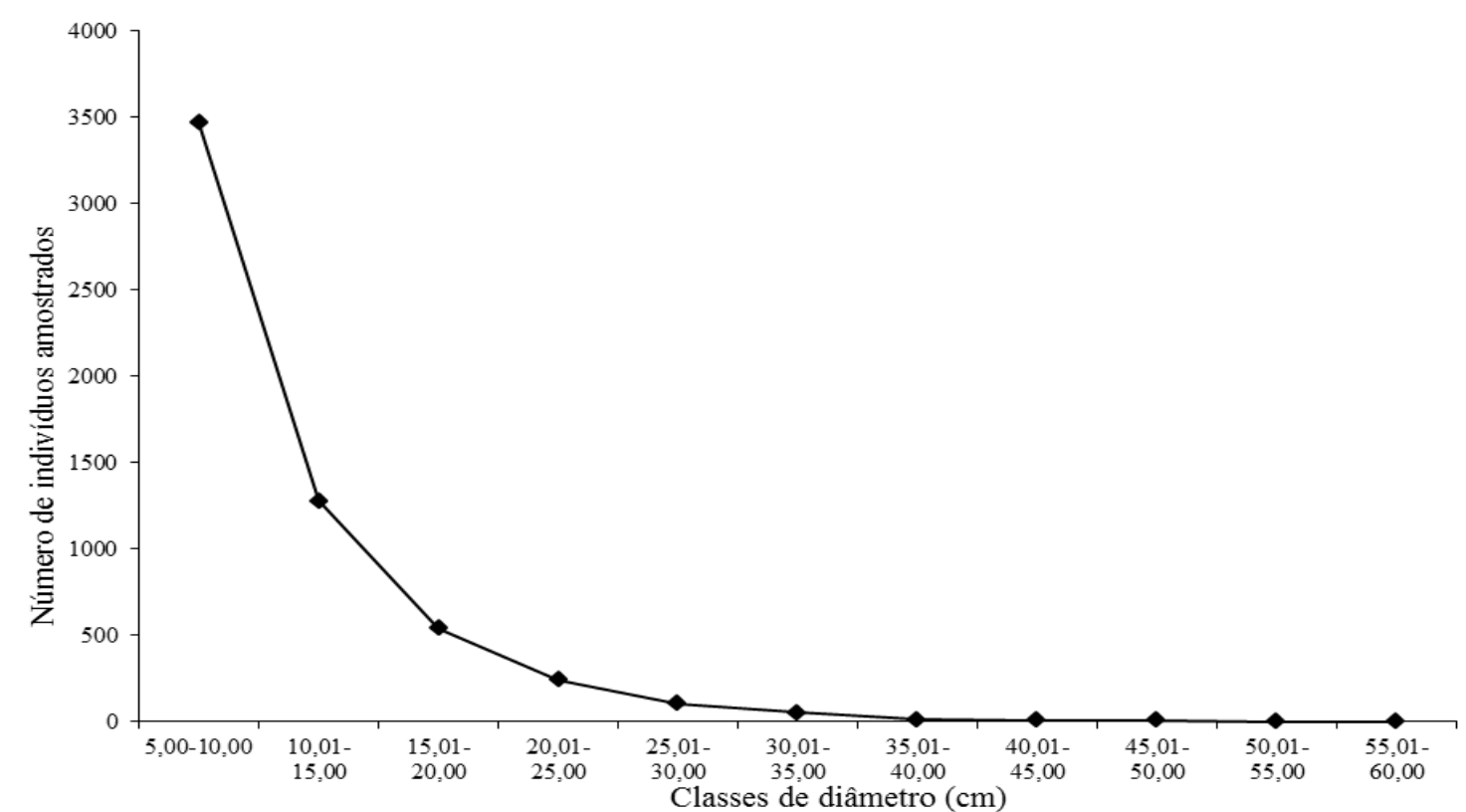

Figura 2. Distribuição de diâmetros de árvores amostradas em cerrado sensu stricto, Mato Grosso, Brasil.

Figure 2. Distribution of diameters of trees sample in cerrado sensu stricto, Mato Grosso, Brazil.

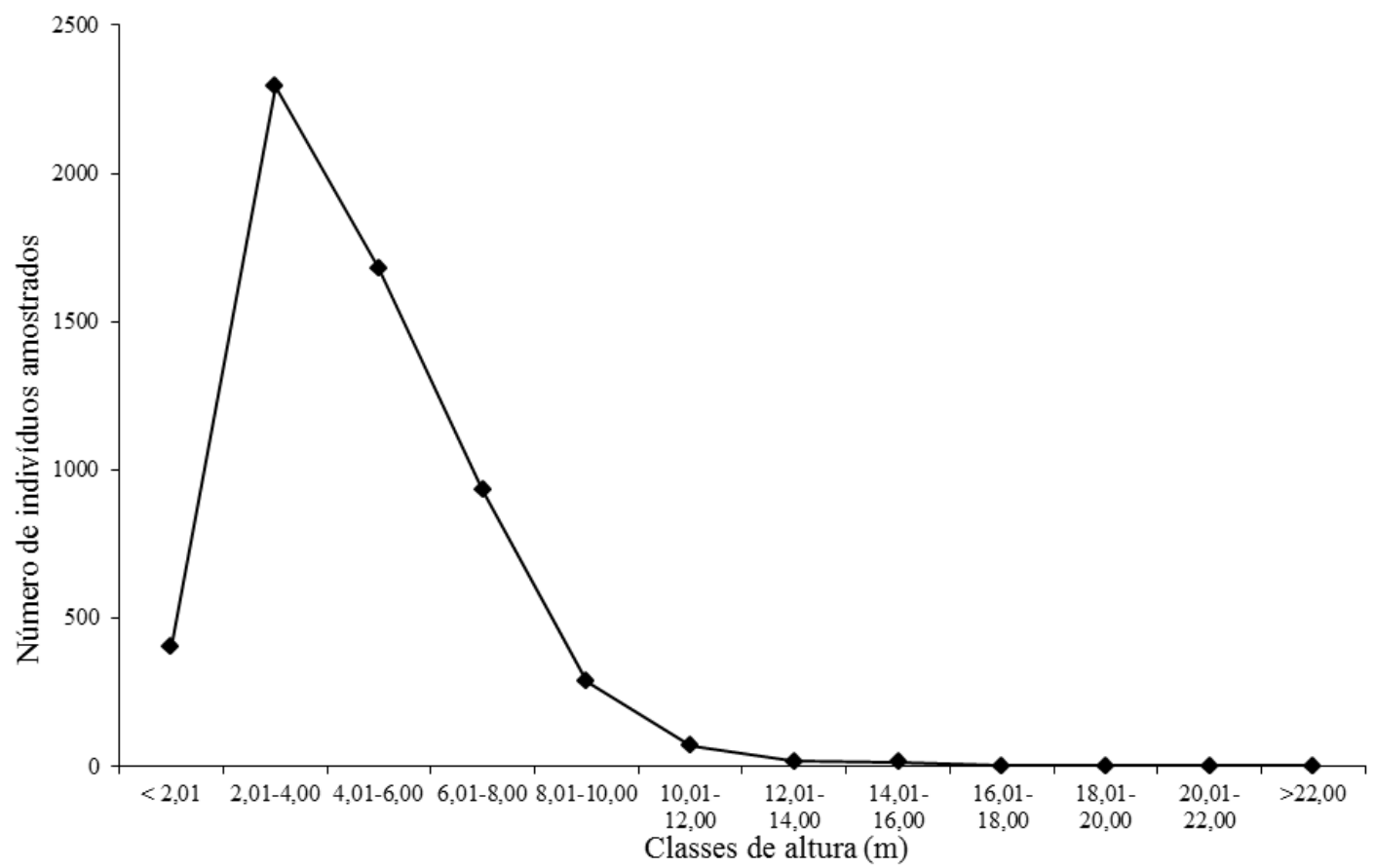

Figura 3. Distribuição de alturas de árvores amostradas em cerrado sensu stricto, Mato Grosso, Brasil. Figure 3. Distribution of heights of trees sample in cerrado sensu stricto, Mato Grosso, Brazil.

A altura total média dos indivíduos amostrados foi estimada em 4,64 m, com um coeficiente de variação das alturas de todos indivíduos amostrados de 47,57\%. O maior número de indivíduos ocorreu 
entre 2,00 e $8,00 \mathrm{~m}$ de altura, que equivale a $88,15 \%$ do total de indivíduos amostrados. Cerca de $6,14 \%$ das espécies encontram-se na classe $2(\mathrm{C} 2)$ de altura $(8,00$ a $6,51 \mathrm{~m}) ; 37,72 \%$ das espécies na classe 3 (C3) de altura $(6,5$ a $5,01 \mathrm{~m}) ; 36,84 \%$ na classe 4 (C4) de altura $(5,00$ a $3,51 \mathrm{~m}) ; 17,54 \%$ na classe 5 (C5) de altura (3,5 a 2,00 m); e apenas uma espécie apareceu na classe $1(\mathrm{C} 1)$ de altura (> 8,00 m) e uma na classe 6 (C6) de altura $(<2,00 \mathrm{~m})$.

Considerando-se as médias dos diâmetros e alturas, as espécies que mais se destacaram foram: Pterodon emarginatus $(d=24,07$ e $h=10,20)$, Dipteryx alata $(d=16,81$ e $h=7,92)$, Bowdichia virgilioides $(d=18,89$ e $h=7,56)$ e Physocalimma scaberrimum $(d=16,87$ e $h=6,90)$. Pterodon emarginatus e Bowdichia virgilioides, quando presentes em fitofisionamias de cerrado sensu stricto, geralmente ocupam o estrato predominante, com copas situando-se sempre acima do dossel $(8,00 \mathrm{~m})$.

Das espécies com maior VI, Pterodon emarginatus pertence à classe $\mathrm{C} 1$ de altura; Terminalia argentea, Magonia pubescens, Hymenaea stigonocarpa, Caryocar brasiliense, Luehea paniculata, Byrsonima pachyphylla, Callisthene fasciculata e Curatella americana pertencem à classe C3; Byrsonima coccolobifolia, Diptychandra aurantiaca, Qualea grandiflora, Couepia grandiflora, Pouteria ramiflora, Qualea parviflora e Lafoensia pacari pertencem à classe C4 e Erythroxylum deciduum, Myrcia albotomentosa e Davilla elliptica pertencem à classe C5, sendo que $92,11 \%$ das espécies amostradas situaram-se nas classes 3, 4 e 5.

As famílias mais bem distribuídas na área estudada foram: Fabaceae e Vochysiaceae (ambas com $F A=98,74)$, que ocorreram em 81 das 82 parcelas levantadas; Dilleniaceae $(F A=91,46)$, amostrada em 75 das 82 parcelas; e Myrtaceae $(F A=90,24)$, presente em 74 das 82 parcelas levantadas.

Apesar de representarem apenas 13,89\% do total de famílias amostradas, mas totalizando $55,36 \%$ da densidade total, as famílias mais bem representadas foram: Vochysiaceae, com 304 indivíduos/ha; Fabaceae, com 246 indivíduos/ha; Dilleniaceae, com 184 indivíduos/ha; Myrtaceae, com 121 indivíduos/ha; e Malpighiaceae, com 106 indivíduos/ha.

Já as famílias que se destacaram em dominância foram: Fabaceae, com $4,75 \mathrm{~m}^{2} / \mathrm{ha}$; Vochysiaceae, com 3,30 m²/ha; Dilleniaceae, com 1,72 $\mathrm{m}^{2} / \mathrm{ha}$; Malpighiaceae, com 1,04 $\mathrm{m}^{2} / \mathrm{ha}$; e Malvaceae, com $0,97 \mathrm{~m}^{2} / \mathrm{ha}$; totalizando $60,01 \%$ da dominância total e apenas $13,89 \%$ do total de famílias amostradas.

As famílias com maior VI e VC foram: Fabaceae, Vochysiaceae, Dilleniaceae, Malpighiaceae, Myrtaceae e Malvaceae. Essas famílias apresentam ampla distribuição nas regiões de cerrados em Mato Grosso e ocorrem com grande freqüência em outros estados da federação, onde essa tipologia se faz representar. Juntas, apesar de representarem apenas $16,67 \%$ do total de famílias amostradas, detiveram $51,99 \%$ do $V I$ e $61,31 \%$ do $V C$.

Os estudos realizados por Gentry et al. (1997) e Mendonça et al. (1998), também confirmaram que as famílias Vochysiaceae, Fabaceae e Myrtaceae são bem representadas nos cerrados do Brasil Central. Um grupo pequeno de famílias prevalecer sobre as demais é uma tendência nas fitofisionomias do tipo cerrado sensu stricto brasileiras. Normalmente as famílias mais uniformemente distribuídas (com maior freqüência absoluta) prevalecem sobre as demais.

\section{Diversidade}

A diversidade alfa da vegetação arbórea, encontrada na área estudada, foi de 4,033 nats/ind., pelo índice de Shannon, e de 0,975, pelo de Simpson, indicando alta diversidade florística, o que é confirmado pelo elevado número de espécies encontradas e pela elevada densidade (Tabela 2). Segundo Pielou (1975) e Martins (1991), os valores de diversidade de Shannon variam de 1,5 a 3,5 nats/ind., raramente passando de 4,5. O índice de Simpson é expresso normalmente como 1-D. A medida que $D$ se incrementa a diversidade decresce.

Esses índices evidenciam que os locais amostrados (em áreas de reserva legal e permanente) apresentam ótimo estado de conservação. A diversidade da área estudada, segundo o índice de ShannonWinner, é equivalente a algumas áreas no Bioma Cerrado, no Brasil Central (Tabela 2). Entretanto, esse índice é alto quando comparado com outras áreas de cerrado sensu stricto.

Possivelmente o contato das comunidades estudadas com fitofisionomias do tipo cerradão, veredas, matas ciliares, matas de galeria e matas de galeria inundáveis, e, ainda, a elevada distância entre grupos de parcelas contribuíram para a riqueza e diversidade de espécies arbóreas verificadas.

FLORESTA, Curitiba, PR, v. 45, n. 4, p. 769 - 780, out. / dez. 2015.

Finger, Z.; Finger, F. A.

ISSN eletrônico 1982-4688 / ISSN impresso 0015-3826 
Tabela 2. Riqueza, densidade, dominância e diversidade: uma comparação entre diversas áreas.

Table 2. Richness, density, dominance and diversity: a comparison among several areas.

\begin{tabular}{|c|c|c|c|c|c|c|c|}
\hline Local* & Riqueza & $\begin{array}{c}\text { Famílias } \\
\left(\mathbf{N}^{\circ}\right)\end{array}$ & $\begin{array}{c}\mathbf{D A}_{\mathbf{i}} \\
\text { (ind/ha) }\end{array}$ & $\begin{array}{c}\operatorname{DoA}_{\mathbf{i}} \\
\left(\mathbf{m}^{2} / \mathbf{h a}\right)\end{array}$ & $\begin{array}{c}\text { Índice } \\
\text { Shannon } \\
\text { (nats/ind.) }\end{array}$ & $\begin{array}{c}\text { Área } \\
\text { amostrada (ha) }\end{array}$ & Referência \\
\hline $\mathrm{CHABAI}^{1}$ & 114 & 36 & 1740 & 19,62 & 4,03 & 3,28 & Presente trabalho \\
\hline $\mathrm{IBGE}^{1}$ & 63 & 34 & 1964 & 13,28 & 3,53 & 0,50 & Andrade et al. (2002) \\
\hline ESEC-AE ${ }^{1}$ & 72 & 31 & 1396 & 10,76 & 3,62 & 6,00 & Felfili e Silva Jr (1993) \\
\hline APA s.s. ${ }^{1}$ & 67 & 32 & 1394 & 10,64 & 3,56 & 6,00 & Felfili e Silva Jr (1993) \\
\hline $\mathrm{PNB}^{1}$ & 55 & 26 & 1036 & 8,32 & 3,34 & 11,00 & Felfili et al. (1997) \\
\hline $\mathrm{PEN}^{1}$ & 52 & 29 & 552 & 7,99 & 3,24 & 1,00 & Rossi et al. (1998) \\
\hline FAL s.s. ${ }^{1}$ & 61 & 30 & 958 & 7,34 & 3,46 & 3,02 & Felfili e Silva Jr (1992) \\
\hline ÁGUA BOA-MT ${ }^{1}$ & 80 & 34 & 995 & 7,45 & 3,69 & 1,0 & Felfili et al. (2002) \\
\hline APA PARANOÁ ${ }^{1}$ & 54 & 30 & 882 & 9,53 & 3,41 & 1,0 & $\begin{array}{c}\text { Assunção e Felfili } \\
\text { (2004) }\end{array}$ \\
\hline REBIOMV s.s. ${ }^{1}$ & 77 & 38 & 1890 & 14,94 & 3,78 & 0,5 & $\begin{array}{c}\text { Marimon Jr. e } \\
\text { Haridasan (2005) }\end{array}$ \\
\hline
\end{tabular}

* CHABAI: (Chapada dos Guimarães e Santo Antônio de Leverger - cerrado sensu stricto); IBGE: (RECOR - cerrado denso); ESEC-AE: (Águas Emendadas - cerrado sensu stricto); APA s.s.: (Gama-Cabeça-do-Veado - cerrado sensu stricto); PNB: (Parque Nacional de Brasília - cerrado sensu stricto); PEN: (Parque Ecológico Norte - cerrado sensu stricto); FAL s.s.: (Fazenda Água Limpa - cerrado sensu stricto); ÁGUA BOA: (Município Água Boa, MT - cerrado sensu stricto); APA PARANOÁ: (Matinha do CO-Brasília, DF - cerrado sensu stricto); REBIOMV s.s.: (Reserva Municipal Mário Viana-Nova Xavantina, MT - cerrado sensu stricto). ${ }^{1}$ Diâmetro dos indivíduos avaliados $\geq 5,00 \mathrm{~cm}$ na base.

\section{CONCLUSÕES}

- As espécies com maior VI foram Qualea parviflora, Curatella americana, Davilla elliptica, Qualea grandiflora, Pterodon emarginatus, Lafoensia pacari, Diptychandra aurantiaca, Myrcia albotomentosa, Caryocar brasiliense, Byrsonima pachyphylla corroborando que em fitofisionomia típica de cerrado sensu stricto a presença de Qualea parviflora, Curatella americana, Davilla elliptica e Lafoensia pacari é comum.

- Os resultados encontrados confirmaram as famílias Fabaceae, Myrtaceae e Vochysiaceae como as mais representativas floristicamente dos cerrados estudados no Brasil Central;

- Um grupo pequeno de espécies e famílias prevaleceu sobre as demais, confirmando ser esta uma tendência nas fitofisionomias cerrado sensu stricto brasileiras. Normalmente as espécies e famílias mais uniformemente distribuídas (com maior frequiência absoluta) são as que prevalecem numa mesma comunidade;

- As comunidades estudadas são constituídas de vegetação com fitofisionomia típica de cerrado sensu stricto, apresentando áreas de contato com fitofisionomias do tipo cerradão, veredas, matas ciliares, matas de galeria e matas de galeria inundáveis, o que pode ser confirmado pela amostragem de algumas espécies (Astronium fraxinifolium, Myracrodruon urundeuva, Tapirira guianensis, Protium heptaphyllum, Copaifera lagsdorffii, Emmotum nitens, Physocalimma scaberrimum, Miconia albicans, Anadenanthera falcata, Plathymenia reticulata, Siparuna guianensis, Dipteryx alata, Machaerium acutifolium, Plathypodium elegans, Zanthoxylum rigidum subsp. hasslerianum, Dilodendron bipinnatum e Guazuma ulmifolia) características dessas fitofisionomias em certas parcelas, que, no processo de dispersão, colonizaram esses habitats;

- A diversidade alfa da vegetação arbórea encontrada na área estudada foi alta (4,033 nats/ind. pelo índice de Shannon), superior à maioria das outras áreas de cerrado com fitofisionomias semelhantes estudadas no Brasil central. Essa elevada diversidade teve certamente como causa principal a presença de áreas de contato com outras fitofisionomias próximas aos fragmentos estudados.

\section{REFERÊNCIAS}

ANDRADE, L. A. Z.; FELFILI, J. M.; VIOLATTI, L. Fitossociologia de uma área de cerrado denso na RECOR-IBGE, Brasília, DF. Acta Botânica Brasílica, São Paulo, v. 16, n. 2, p. 225 - 240, 2002. 
ANGIOSPERM PHYLOGENY GROUP III (APG III). An update of the Agiosperm phylogeny group classification for the orders and families of flowering plants: APG III. Annals of the Botanical Journal of the Linnean Society, v. 161, n. 2, p. 105 - 121, 2009.

ASSUNÇÃO, S. L.; FELFILI, J. M. Fitossociologia de um fragmento de cerrado sensu stricto na APA do Paranoá, DF, Brasil. Acta Botânica Brasílica, São Paulo, v. 18, n. 4, p. 903 - 909, 2004.

BORLAUG, N. E. Freeding a world of 10 billion people: the miracle ahead. In: BAYLEY, R. (Ed.). Global warming and other eco-myths. Roseville-EUA: Competitive Enterprise Institute, 2002. p. 29 - 60.

COUTINHO, L. M. Fire in the ecology of the brazilian cerrado. In: GOLDAMMER, J. G. Fire in the tropical biota: ecosystem processes and global challenges. Berlin: Springer-Verlag, 1990., p. 82 - 103.

COLWELL, R. K. EstimateS 8.2.0. Statistical estimation of species richness and shared species from samples. Version 8.2.0. http://viceroy.eeb.uconn.edu/EstimateSPages/EstimateS.flx (último acesso em 21/10/2011).

EMPRESA BRASILEIRA DE PESQUISA AGROPECUÁRIA (EMBRAPA). Sistema brasileiro de classificação de solos. Rio de Janeiro: Empresa Brasileira de Pesquisa Agropecuária, Centro Nacional de Pesquisa de Solos. 360 p. 1999.

FELFILI, J. M. Diameter and height distributions in a gallery forest tree community and some of its main species in central Brazil over a six-year period (1985-1991). Revista Brasileira de Botânica, São Paulo, v. 2, p. 155 - 162, 1997.

FELFILI, J. M.; NOGUEIRA, P. E.; SILVA JR., M. C.; MARIMON, B. S.; CARVALHO DELITTI, W. B. Composição florística e fitossociológica do cerrado sentido restrito no município de Água Boa, MT. Acta Botânica Brasílica, v. 16, n. 1, p. 103 - 112, 2002.

FELFILI, J. M.; FILGUEIRAS, T. S.; HARIDASAN, M.; SILVA JUNIOR, M. C.; MENDONÇA, R. C.; REZENDE, A. V. Projeto biogeografia do bioma cerrado: vegetação e solos. Cadernos de Geociências, v. 12 , n. 4, p. 75 - 166, 1994.

FELFILI, J. M.; SILVA JR., M. C. Distribuição dos diâmetros numa faixa de cerrado na Fazenda Água Limpa, Brasília, DF. Acta Botanica Brasílica, São Paulo, v. 2, p. 85 - 104, 1988.

FELFILI, J. M.; SILVA JR., M. C. Floristic composition, phytosociology and comparison of cerrado and gallery forests at Fazenda Água Limpa, Federal District, Brazil. In: FURLEY, P. A.; PROCTOR, J. A.; RATTER, J. A. (Eds.). Nature and dynamics of forest-savanna boundaries. London: Chapman and Hall, 1992. p. 393 - 615.

FELFILI, J. M.; SILVA JR., M. C. A comparative study of cerrado sensu strictu vegetation in Central Brasil. Journal of Tropical Ecology, Cambridge, v. 9, p. 277 - 289, 1993.

FURLEY, P. A.; RATTER, J. A.; GIFFORD, D. R. Observations on the vegetation of esatern Mato Grosso, Brazil III. The woody vegetation and soils of the Morro da Fumaça, Torixoréu. Phil. Trans. R. Soc. Lond. London, v. 235, p. 259 - 280, 1988.

GENTRY, A. H.; HERRERA-MAC BRYDE, O.; HUBER, O.; NELSON, B. W.; VILLAMIL, C. B. Regional overview: South America. In: HEIWOOD, V. H.; DAVIS, S. D. (Eds.). Centres of plant diversity. Cambridge: WWF/IUCN, 1997. p. 269 - 307.

INSTITUTO BRASILEIRO DE GEOGRAFIA E ESTATÍSTICA/INSTITUTO BRASILEIRO DE DESENVOLVIMENTO FLORESTAL (IBGE/IBDF). Mapa de vegetação do Brasil (1:5.000.000). Rio de Janeiro: IBGE, 1993.

KENT, M.; COKER, P. Vegetation description analyses. London: Behaven, 1992. 363 p.

MARIMON, B. S.; VARELLA, R. F.; MARIMON JR., B. H. Fitossociologia de uma área de cerrado de encosta em Nova Xavantina, Mato Grosso. Boletim do Herbário Exechias Paulo Heringer, Brasília, v. 3, p. 82 - 101, 1998.

FLORESTA, Curitiba, PR, v. 45, n. 4, p. 769 - 780, out. / dez. 2015. 
MARIMON JR., B. H.; HARIDASAN, M. Comparação da vegetação arbórea e características edáficas de um cerradão e um cerrado sensu stricto em áreas adjacentes sobre solo distrófico no leste de Mato Grosso, Brasil. Acta Botanica Brasílica, São Paulo, v. 19, n. 4, p. 913 - 926, 2005.

MARTINS, F. R. Estrutura de uma floresta mesófila. São Paulo: Ed da UNICAMP, 1991. 246 p.

MENDONÇA, R. C.; FELFILI, J. M.; WALTER, B. M. T.; SILVA JÚNIOR, M. C.; REZENDE, A. V.; FILGUEIRAS, T. S.; NOGUEIRA, P. E. Flora vascular do cerrado. In: SANO, S. M. e ALMEIDA, S. P. (eds.). Cerrado: ambiente e flora. Planaltina-DF: Embrapa- CPAC, 1998. p. 287 - 556.

MUELLER-DOMBOIS, D.; ELLENBERG, H. Aims and methods of vegetation ecology. New York: John Wiley \& Sons, 1974. $547 \mathrm{p}$.

PEREIRA JR., A. C. Monitoramento de queimadas na região dos cerrados utilizando dados AVHRR/NOAA corrigidos por dados TM/Landsat. 1992. 268 f. Dissertação (Mestrado em Sensoriamento Remoto) - Instituto Nacional de Pesquisa Espacial, São José dos Campos, 1992.

PIELOU, E. C. Ecological diversity. New York: John Wiley, 1975. 165 p.

PIRES, A.; FELFILI, J. M.; RIBEIRO, A. A. Florística e fitossociologia do cerrado sensu stricto da APA do Cafuringa. Boletim do Herbário Ezechias Paulo Heringer, Brasília, v. 4, p. 5 - 20, 1999.

RATTER, J. A.; BRIDGEWATER, S.; ATKINSON, R.; RIBEIRO, J. F. Analysis of the floristic composition of the brazilian cerrado vegetation II: Comparison of the woody vegetation of 98 areas. Edinburgh Journal of Botany, v. 53, n. 2, p. 153 - 180, 1996.

ROSSI, C. V.; SILVA JR., M. C.; SANTOS, C. E. N. Fitossociologia do estrato arbóreo do cerrado "sensu stricto" no Parque ecológico Norte, Brasília, DF. Boletim do Herbário Ezechias Paulo Heringer, Brasília, v. 2, p. 49 - 56, 1998.

SANO, M. S.; DE ALMEIDA, S. P.; RIBEIRO, J. F. Cerrado: Ecologia e Flora. v. 2, Brasília: Embrapa Informação Tecnológica, 2008. 1.279 p. 